\title{
A VLA GRAVITATIONAL LENS SURVEY
}

J. N. Hewitt (MIT and Haystack Observatory), E. L. Turner (Princeton), B. F. Burke (MIT), C. R. Lawrence (Caltech), C. L. Bennett (NASA/GSFC), G. I. Langston (MIT), J. E. Gunn (Princeton)

Gravitational lens surveys are of cosmological interest because they provide a way to measure the gravitational field of both luminous and dark matter. Many of the other methods used to detect the presence of dark matter, such as studies of galaxy rotation curves and cluster dynamics, require that there be luminous objects in the gravitational field that act as tracers of the mass. This may introduce a selection effect. In constrast, in studies of gravitational lenses, the beacon we observe can be far (at distances of order one thousand Mpc) from the gravitational field. In this paper we describe a VLA survey designed to detect gravitational lensing on sub-arc second and arc second scales. We also present a preliminary result of the radio data: we find that the density of matter in the form of a uniform, comoving number density of $10^{11}$ to $10^{12} M_{\odot}$ compact objects, luminous or dark, must be substantially less than the critical density.

The potential of a gravitational lens survey as a cosmological tool motivated our VLA survey. The strategy of the lens survey is as follows. First we map many MG (Bennett, et al. 1986) sources with the VLA, and select lens candidates on the basis of radio morphology. These candidates are subjected to lens tests, for example: (1) Optical imaging - do the candidate images have similar optical and radio flux ratios? (2) Optical spectroscopy - do the candidate images have the same redshift and similar spectra? (3) VLBI - do the candidate images have milli-arc second radio morphology consistent with gravitational imaging? We are carrying out a program of optical verification of lens candidates, and some preliminary VLBI observations are planned. To our knowledge, this is the first time a large set of radio data has been collected systematically, with a good understanding of the instrumental selection biases, for the purpose of studying the properties of lensed sources.

The VLA has well defined instrumental limits that determine its ability to detect gravitational lens systems. The resolution and field of view limit the range of image separations detectable; the dynamic range limits the range of flux ratios detectable. For a given lens system, characterized by its total mass, mass distribution, and the distances to the lens and the source, the instrumental limits can be mapped into a corresponding range of source positions that produce detectable multiple images. We then assume a spatial distribution of lenses and treat the source position as a random variable within the range determined by the instrumental limits. This allows one to calculate the expected frequency of lensing and the properties of lensed images in a sample of radio sources. This method is similar 
to that of Turner, Ostriker, and Gott (1984); it differs in that we have included the effects of known instrumental limits.

The frequency of lensing in a sample of radio sources provides a measurement of $\Omega_{L}$, where $\Omega_{L}$ is the density of matter in the form of lenses expressed as a fraction of the critical density. For example, in the case of point mass lenses, the cross section for the formation of multiple images scales with the mass $M$; the number density of lenses scales with $\Omega_{L} / M$; and the frequency of lensing scales with just $\Omega_{L}$ (Press and Gunn 1973). If we calculate the optical depth to lensing (which for small optical depths gives the probability of lensing) for a uniform comoving distribution of point mass lenses, including the instrumental limits of the VLA in the A array, we find the results plotted in Figure 1. The VLA in the A array at an observing wavelength of $6 \mathrm{~cm}$ is sensitive to $10^{11}$ to $10^{12} M_{\odot}$ lenses. We can use the radio data to constrain $\Omega_{L}$. As with the microwave background, one thing that is interesting is what we do not see. We can put limits on the density of matter in the form of compact lenses by counting the number of sources that are not lensed on the scales detectable with the VLA. To relate this to $\Omega_{L}$, we will make a few conservative assumptions.

First, we ignore the effect of a flux intensification bias on the sample. This bias causes more lensed images to be included in any flux limited sample. Second, we assume the redshift distribution of the sources is the same as that measured for 30 optically bright sources (to the extent that faint sources have the same redshift distribution as bright sources, as suggested at this conference by Koo, this assumption may not be conservative). Third, we make the ridiculously conservative assumption that any structure in a VLA map may be due to gravitational lensing. Then, for a sample of 290 sources observed at a wavelength of $6 \mathrm{~cm}$ with the A array of the VLA, we count the number of sources that could be lensed. The maximum value of $\Omega_{L}$ consistent with the data and a background metric described by $\Omega=1$ (again a conservative assumption in the sense that it gives a large value of $\Omega_{L}$ ), for masses in the range of $10^{11}$ to $10^{12} M_{\mathcal{O}}$, is 0.7 .

We can modify the third assumption by assuming, perhaps more reasonably, that resolved, collinear components represent intrinsic source structure and not lens effects. Then we find that, again for masses in the range of $10^{11}$ to $10^{12} M_{\odot}$, the maximum consistent value of $\Omega_{L}$ is 0.4 . These maximum values of $\Omega_{L}$ are independent of the value of $H_{o}$.

In summary, even at this very early stage in our gravitational lens survey, the data strongly suggest that one cannot close the universe with luminous or dark galaxy-sized compact objects. We expect that future work will further constrain $\Omega_{L}$. The observations will continue to eliminate lens candidates, and the inclusion in the calculations of the flux intensification bias will decrease the maximum density of lenses that is needed to explain a given frequency of lensing.

Finally, to demonstrate that we are not only eliminating lens candidates, but we are also finding some interesting ones, we present the radio source $1042+178$ in Figure 2. This source, with four components arranged in a parallelogram, has extremely unusual radio morphology. An optical object at the position of the radio source appears to have some structure. A spectrum of the combined light from the source shows strong emission lines at a redshift of 0.921 . If this source is lensed, the lensing may well be due to a single galaxy. Such a simple mass distribution, combined with the low redshift of the source that reduces cosmological distance uncertainties, may make this a good system to use to measure the Hubble constant through a measurement of the time delay. 
Figure 1: Relative sensitivity of the VLA observations to compact gravitational lenses of different masses, for three values of the source redshift. A curve has been drawn through numerically calculated points to guide the eye.

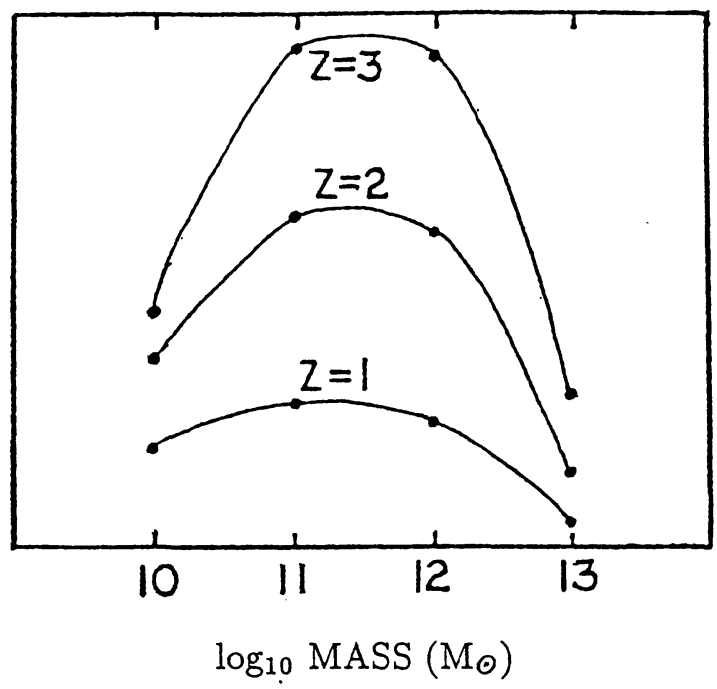

Figure 2: VLA map (A array; $\lambda=6 \mathrm{~cm}$ ) of $1042+178$.

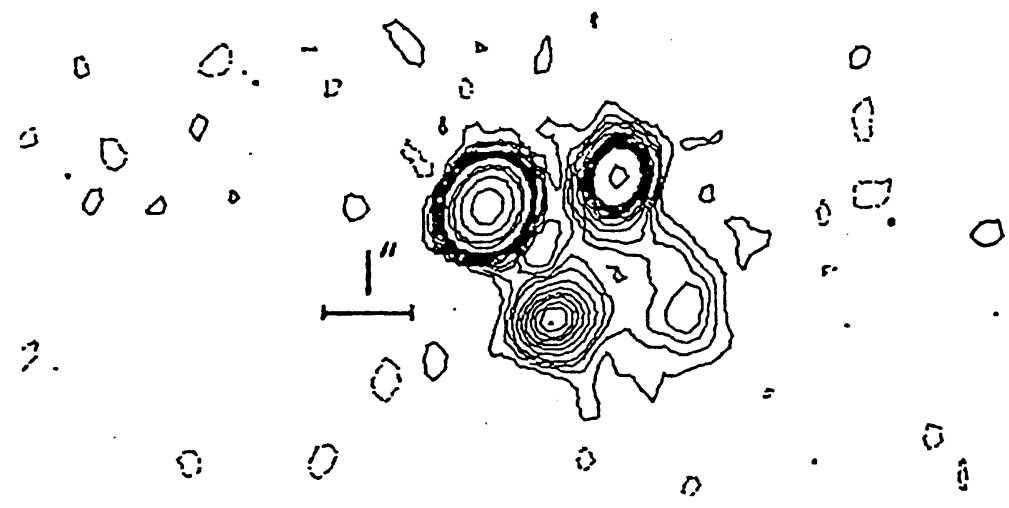

This research was supported in part by grants AST-8415677 and AST:-8512598 from the National Science Foundation.

\section{REFERENCES}

Bennett, C. L., Lawrence, C. R., Burke, B. F., Hewitt, J. N., and Mahoney, J. H. 1986, Ap. J. (Supp.), 61, 1 .

Press, W. H., and Gunn, J. E. 1973, Ap. J., 185, 397.

Turner, E. L., Ostriker, J. P., and Gott, J. R., III. 1984, Ap. J., 284, 1. 


\section{DISCUSSION}

PECKER: How would your hypothesis that most radio structure of radio sources (or at least many of them) is due to lensing effects, affect the $\theta-Z$ relation (the classical Hubble Tolman test)?

HEWITT: A flux-limited sample would preferentially select lensed sources because of the flux intensification effect. Therefore, I would expect that the focussing due to the "extra" matter along the line of sight would tend to cause an increase in $\theta$ with increasing redshift. I would like to note that my "hypothesis" that most radio structure is due to lensing was a devil's advocate assumption I made to find a conservative limit on the density of matter in the form of compact masses. 